\title{
O emprego da ANÁlise de Risco como FERRAMENTA DA INTELIGÊNCIA ESTRATÉGICA
}

\author{
Carlos eduardo pires de albuquerque \\ E FELIPE SCARPELLi de ANDRADE \\ Departamento de Polícia Federal - Brasil

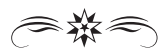

\begin{abstract}
RESUMO
O presente trabalho apresenta uma proposta para aprimorar o assessoramento ao processo decisório por meio do emprego da análise de risco como ferramenta de inteligência estratégica, de maneira conjugada com técnicas prospectivas, trazendo uma apresentação do contexto atual e de sua aplicabilidade.
\end{abstract}

Palavras-Chave: Inteligência. Análise de risco. Inteligênvcia estratégica.

\section{INTRODUÇÃ̃o}

Os dias atuais carregam em si a incerteza resultante da quantidade de informações disponíveis para amparar decisões nos mais diversos níveis organizacionais. A integração dessas informações e a produção de um conhecimento mais confiável são fatos necessários para permitir que sejam tomadas decisões embasadas, garantindo segurança aos tomadores de decisão e às organizações no seu desenvolvimento presente e futuro.

Nesse contexto, surge a análise de risco (AR), técnica capaz de analisar cenários com potenciais ameaças e oportunidades, proporcionando às organizações a capacidade de agir preventivamente através do planejamento de suas ações no sentido de mitigar perdas ou aproveitar cenários favoráveis. Por meio da compreensão da natureza do risco e da determinação de seu nível, baseado em critérios de probabilidade e da estimativa dos impactos resultantes do acontecimento de um evento, é possível apresentar ações, tendo como base os Cenários Prospectivos", que mitiguem os riscos inerentes e auxiliem na condução de resultados, de forma a subsidiar a tomada de decisão. 
O emprego da análise de risco como ferramenta da inteligência estratégica

De acordo com Schwartz (2000), cenários prospectivos são histórias sobre a forma que o mundo pode assumir amanhã, são quadros capazes de nos ajudar a reconhecer as possíveis mudanças de nosso ambiente globalizado e como nos adaptarmos a essas transformações. Portanto, trata-se de uma importante técnica que descortina nossas visões sobre os factíveis e mais prováveis acontecimentos futuros, de acordo com o cenário presente.

Os cenários prospectivos emergem também na atualidade, com capacidade de auxiliar na construção de um futuro melhor, integrando o planejamento estratégico das instituições, e auxiliando a chegada a um futuro desejado, afastando eventos não desejáveis e aproveitando os que interessem à instituição.

O presente trabalho tem por objetivo apresentar as linhas gerais acerca da AR como técnica acessória de inteligência no assessoramento estratégico ao processo decisório, de maneira conjugada com técnicas prospectivas de construção de cenários.

\section{ANÁlise de Risco}

\section{2.i. Contexto Atual}

Atualmente, dentro do contexto das organizações, as decisões cercam-se de incerteza em face do volume de informações disponíveis, sendo necessário garantir a segurança na tomada da decisão visualizando os riscos inerentes.

Risco é o efeito da incerteza nos objetivos. Um efeito é um desvio em relação ao esperado - positivo elou negativo. A incerteza é o estado, mesmo que parcial, da deficiência das informações relacionadas a um evento, sua compreensão, conhecimento, sua consequência ou probabilidade. (ISO GUIDE 73:2009, 2009, p. 1).

A Gestão de Riscos (GR), ou Gerenciamento de Riscos, encontra-se na vanguarda das práticas corporativas, sendo uma tendência mundial para o assessoramento estratégico ao decisor nas mais diversas áreas de atuação, tanto no campo privado como no governamental. 
Segundo o Department of Homeland Security $\left(D H S^{1}\right)$, o gerenciamento de riscos é o processo de identificação, análise, avaliação e comunicação dos riscos, através da sua aceitação, evitação, transferência ou controle a um nível aceitável, considerando os custos associados e benefícios de quaisquer ações tomadas. (EUA, 2011, p. 7). A GR surgiu de maneira eficiente agregando valor no assessoramento ao processo decisório.

Atualmente, há diversas agências oficiais que utilizam análises de riscos voltadas ao assessoramento estratégico, em temas como: combate ao crime organizado e ao terrorismo, proteção de infraestruturas críticas, eventos nacionais, inteligência e investigação, dentre outros focos, como a Australian Crime Comission $(A C C)^{2}$, a Serious Organized Crime Agency $(S O C A)^{3}$ e o $D H S$. No Brasil, de forma geral, essa técnica é utilizada com viés de segurança orgânica e instalações físicas.

Cabe destacar o prefácio da Estratégia Nacional de Contrainteligência dos Estados Unidos que explicita que "os objetivos estratégicos essenciais não devem mudar a cada ano; no entanto o processo de gestão de riscos exige que a Comunidade de Contrainteligência atue continuamente a avaliar vulnerabilidades, oportunidades e desafios". (EUA, 2007, p. vi).

O DHS estabeleceu em 2010 uma Política para Gestão Integrada de Riscos, tendo, como ponto central, o esforço que deve ser de todos os parceiros de segurança interna em nível federal, estadual, local, tribal, territorial, não governamental, e também do setor privado. Em 2011, lançou uma publicação denominada "Fundamentos do Gerenciamento de Riscos - Doutrina de Gerenciamento de Riscos da Segurança Interna”, cumprindo um papel essencial na condução do esforço unificado para controlar os riscos para os Estados Unidos da América, a partir de um conjunto diversificado e complexo de ameaças, incluindo os atos de terrorismo, as catástrofes naturais e de origem humana, as pandemias, os ataques cibernéticos e a criminalidade transnacional. (EUA, 2011, p. 1).

1 Responsável pelo Gerenciamento de Riscos de Infraestruturas Críticas, Autoridade e de Eventos Nacionais nos Estados Unidos, efetivando inclusive convênios com Universidades para criação do National Center for Risk and Economic Analysis of Terrorism Events - CREATE.

$2 \mathrm{O} A C C$ substituiu a Autoridade Nacional de Crime, a Agência Australiana de Inteligência Criminal $(A B C I)$, e o Escritório de Avaliações Estratégicas de Crime para se tornar a Agência Nacional de Inteligência Criminal e Investigação da Austrália.

3 Atua dentro da doutrina governamental inglesa de gestão de riscos, desenvolvida a partir do Modelo de Excelência da EFQM, tendo foco em multiameaças, como lavagem de dinheiro e financiamento do terrorismo. 
O emprego da análise de risco como ferramenta da inteligência estratégica

No Brasil, destaca-se a atuação da Agência Brasileira de Inteligência (Abin) que, desde 2005, desenvolve a Metodologia ARENA (Análise de Riscos com Ênfase na Ameaça), como atribuição do Departamento de Contrainteligência (DCI/Abin), possuindo três vertentes de elaboração de Análises de Riscos: Segurança da Informação, Proteção de Infraestruturas Críticas e Grandes Eventos. As Forças Armadas brasileiras também têm empregado a AR, com foco na segurança orgânica, no ramo de Contrainteligência.

O Departamento de Polícia Federal está desenvolvendo o Método Sabre, por intermédio do Serviço de Análise Estratégica e Estatística da Diretoria de Inteligência Policial (SAEE/DIP/DPF), orientado para as suas Atribuições Constitucionais, em atendimento a demanda do Planejamento Estratégico e Gerenciamento de Projetos ligados aos Grandes Eventos que ocorrerem no Brasil.

\subsection{DEFINIÇÕES}

Antes de se definir a AR, cabe destacar a diferença entre risco e problema, que muitas vezes são considerados como sinônimos. Aquele, é um evento incerto, que pode vir a ocorrer, enquanto este, trata-se de um fato indesejado, que já ocorreu. Outro conceito que deve ser claro é o de proprietário do risco, que representa a pessoa ou entidade com a responsabilidade e a autoridade para gerenciar o risco. (ISO GUIDE 73:2009, 2009, p. 2).

A doutrina do $D H S$ explicita um processo de GR que apresenta as seguintes fases: A definição do contexto - definição e elaboração do contexto das decisões, metas relacionadas e os objetivos; A identificação de riscos potenciais - identificação dos riscos associados com as metas e objetivos; A avaliação e análise do risco - avaliação e análise dos riscos identificados; $\mathrm{O}$ desenvolvimento de alternativas - desenvolvimento de ações alternativas para a gestão dos riscos e a criação de oportunidades, e análise dos seus custos e benefícios; A decisão e execução - tomada de decisão entre linhas de ação e executar essa decisão; e a avaliação e monitoramento - acompanhamento da decisão tomada, comparando-se os efeitos da ação decorrente aos efeitos observados e esperados, para ajudar a influenciar decisões futuras. (Figura 1). 


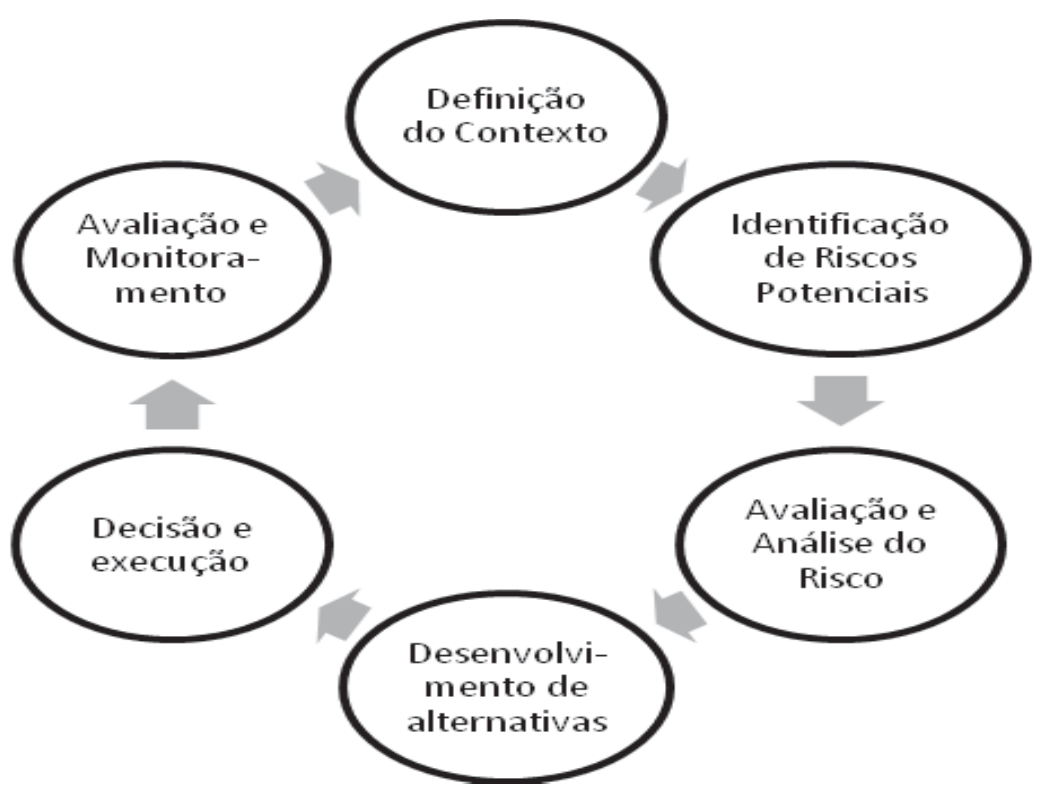

Figura 1 Processo de Gerenciamento de Riscos do DHS. Fonte: EUA (2011, p. 12).

\subsection{APLICABILIDADES DA ANÁLISE DE RISCOS}

A AR não deve ser um fim em si mesma, devendo ser incluída nas práticas corporativas que incluam o planejamento, a preparação, a avaliação do programa, a melhoria de processos e o desenvolvimento de prioridade orçamentária. $\mathrm{O}$ valor de um trabalho de GR apresentado aos tomadores de decisão não se encontra no levantamento de uma determinada linha de ação, mas sim na capacidade de se distinguir entre diversas opções em um contexto mais amplo. (EUA, 2011, p. 8).

A AR é fundamental para os decisores, pois amplia a capacidade em priorizar requisitos concorrentes, proporcionando uma abordagem abrangente para acompanhar o desempenho e detalhamento do progresso de ações. (EUA, 2011, p. 8).

As práticas da GR permitem uma abordagem sistemática para assessorar a tomada de decisão, promovendo o seu desenvolvimento e a sua utilização em questões como a Segurança Pública e Segurança Nacional, apresentando a melhor seleção entre estratégias e ações alternativas, e avaliando a eficácia das atividades que realizam. 
O emprego da análise de risco como ferramenta da inteligência estratégica

As aplicações da AR incluem o Planejamento Estratégico, projetando estratégias em uma visão de longo prazo para criação de capacidades que podem mitigar os riscos através da prevenção, da proteção, das respostas e de atividades de recuperação, moldando as organizações com abordagens de construção e sustentação de capacidades. A análise de risco pode ser empregada no planejamento baseado em capacidades, identificando quais são os recursos mais importantes para uma organização e quais são as lacunas de capacidades potenciais. (EUA, 2011, p. 9).

Considerando a necessidade de tomada de decisão executiva e estratégica pelos decisores, a técnica de AR pode auxiliá-los continuamente no monitoramento e em ações em curso, proporcionando uma visão do contexto do risco das diferentes ameaças e perigos para estruturas estratégicas, áreas geográficas e centros populacionais durante situações de crise. (EUA, 2011, p. 9).

Cabe destacar que um relatório de AR não possui força executória ou mandatória, não é exaustivo, sendo um instrumento de assessoramento estratégico ao planejamento e ao processo decisório para os mais altos escalóes da organização.

A Gestão dos Riscos assume um papel de destaque dentre as modernas técnicas corporativas, principalmente, se associada a técnicas prospectivas, permitindo o monitoramento de ameaças reais ou potenciais identificadas, além de proporcionar sustentação ao desenvolvimento de ações estratégicas, ressaltando inclusive oportunidades.

\section{INTELIGÊNCIA ESTRATÉgICA}

\section{I CONCEITOS E DEFINIÇÕES}

De acordo com Cepik (2001, p. 24), na acepção ampla, afora a definição da ciência cognitiva, Inteligência é o conhecimento ou informação analisada. A inteligência, portanto, é definida como a atividade que visa assessorar autoridades governamentais no planejamento, execução e acompanhamento das políticas de estado.

A Lei no. 9.883, de 7 de setembro de 1999, instituiu o Sistema Brasileiro de Inteligência (Sisbin) e criou a Agência Brasileira de Inteligência 
(Abin) com o objetivo de integrar as ações de planejamento e execução das atividades de inteligência do País, com vistas a subsidiar o Presidente da República nos assuntos de interesse nacional.

O conceito e a dimensão da atividade de inteligência estão previstas no art. $2^{\circ}$, do Decreto $n^{\circ}$. 4.376/2002, que dispõe sobre a organização e o funcionamento do Sistema Brasileiro de Inteligência:

Art. 2- Para os efeitos deste Decreto, entende-se como inteligência a atividade de obtenção e análise de dados e informaçôes e de produção e difusão de conhecimentos, dentro e fora do território nacional, relativos a fatos e situações de imediata ou potencial influência sobre o processo decisório, a ação governamental, a salvaguarda e a segurança da sociedade e do Estado.

No que tange às atividades de inteligência, é fundamental a troca de informações entre as estruturas que realizam esse tipo de atividade. Portanto, faz-se necessário estabelecer uma relação para que atuem de maneira sistemática na necessária produção de conhecimentos voltados ao estabelecimento de estratégias ou adoção de medidas obstativas a fatores adversos à sociedade e ao Estado.

A AR é uma importante ferramenta nesse contexto, pois se trata de um estudo técnico aprimorado das ameaças futuras, aplicando-se fórmulas de mensuração a partir de um levantamento pré-determinado, como, por exemplo, de uma estrutura física, negócio, bens patrimoniais, atribuições ou pessoas, no sentido de identificar vulnerabilidades e apontar ações que possam mitigar esse risco.

Nessa esteira, a AR torna-se fundamental para a tomada de decisão, pois é uma importante forma de se estabelecer a consecução desse propósito.

\subsection{A UTILIZAÇÃO DA ANÁLISE DE RISCO COMO TÉCNICA ACES- SÓRIA AO PROCESSO DECISÓRIO}

$\mathrm{Na}$ conjuntura das atividades de inteligência, o conhecimento e a sua gestão passam a ter papel relevante, pois qualificam a informação e se apresentam como forma que permite diminuir incertezas, ampliar as possibilidades dos processos decisórios e potencializar as ações e estratégias organizacionais e operacionais. 
O emprego da análise de risco como ferramenta da inteligência estratégica

[...] a gestão da informação e a gestão do conbecimento atuam objetivando diminuir situações ambiguas e com alto indice de incerteza, possibilitando às pessoas da organização o acesso e o uso de informações que agirão sobre essas situações. O nivel de complexidade da situação requer atenção quanto à precisão, relevância e propósito da informação. $O$ ambiente organizacional complexo necessita de condições que amenizem a complexidade dos processos existentes neste âmbito, e a informação é um elemento que proporciona a diminuição dessa complexidade. Para isso é necessário que as organizaçôes possuam um setor especializado que vise à gestão, organização, tratamento e disseminação da informação. (VALENTIM, 2008, p. 23-24).

A era da informação e do conhecimento exige que a sociedade e suas organizações ampliem suas estratégias e ações visando à construção de um ambiente social equilibrado e com o mínimo de conflitos, e as atividades de inteligência são fundamentais nesse processo, pois não basta a intuição e o mero uso da força, mas, sim, o aproveitamento das vantagens do conhecimento e de sua adequada gestão para o seu enfrentamento.

A AR em conjunto com a atividade de inteligência visa, entre outros fatores, interpretar o ambiente complexo da organização e pode ser descrita como:

[...] um modelo mental no qual se baseiam os processos de relacionamento entre organização e ambiente; ter arquitetura e plataformas tecnológicas, melhorar o desempenho da organização de forma global em sintonia com conhecimento pertinente. É a capacidade de julgamento de um problema que surge pelo conhecimento distribuido na organização, com vistas à utilização na consecução de seus objetivos e como principal meta de apoio ao processo decisório em todos os niveis. (FERRO JUNIOR, 2008, p. 97).

Os problemas de tomadas de decisão são constantes no dia-a-dia e a todo o momento as pessoas estão sendo colocadas em uma posição em que é necessário examinar, investigar, decidir, escolher e agir frente às poucas ou muitas opções que lhes são fornecidas. A finalidade da inteligência é justamente esse, assessorar por meio de informações relevantes, o tomador de decisões.

Atualmente, emerge uma nova forma de pensar em relação à tomada de decisão. Aos tomadores de decisão, é dado o desafio de pensar e usar, em larga escala, os instrumentos de informação e comunicação que venham a colaborar no processo decisório. 
Nesse sentido, torna-se imperioso aprimorar a produção de conhecimento em nível estratégico, como fator decisivo para o assessoramento dos tomadores de decisão, com vistas a subsidiar a adoção de medidas que resultem no adequado combate às ameaças dentro de um contexto mais amplo.

Faz-se necessário, então, organizar e estruturar mecanismos para prover alguns tipos específicos de informação. A ferramenta de AR vem ao encontro desse entendimento, possibilitando um melhor planejamento para a tomada de decisão.

Portanto, é fundamental para um procedimento de decisão adequado, iniciar, por meio de técnicas especializadas de processamento de informações, a identificação das necessidades, do que é possível fazer, da informação que está disponível, e da comunicação que precisa ser efetuada. Espera-se que estes elementos resultem na possibilidade de assessorar com informações oportunas uma melhor tomada de decisão. Nesse sentido, leciona Ribeiro (2003, p. 101):

O tomador de decisöes, quer esteja motivado pela necessidade de prever ou controlar, geralmente enfrenta um complexo sistema de componentes correlacionados, como recursos, resultados ou objetivos desejados, pessoas ou grupos de pessoas; ele está interessado na análise desse sistema. Presumivelmente, quanto melhor ele entender essa complexidade, melhor será sua decisão.

Segundo Simon (1963, p. 115), a decisão é um processo de análise e escolha entre várias alternativas disponíveis do curso de ação a ser seguida.

Drucker (1972, p. 157) denomina as decisões de táticas e estratégicas. As decisões táticas são mais simples, podendo-se confiar na capacidade intuitiva do decisor de tomar a decisão acertada, enquanto que as decisões estratégicas são mais difíceis, pois o problema e a solução são desconhecidos, fazendo com que a tomada de decisão seja sempre obtida através de um processo visando à solução do problema.

Conforme exposto, a inteligência tem por finalidade auxiliar a decisão e a AR se apresenta como uma importante ferramenta nesse processo, pois permite identificar quais as maiores ameaças e vulnerabilidades com que o decisor ira se deparar.

A atividade de inteligência, quando auxiliada pela AR, pode assessorar o gestor, no sentido de melhor empregar os recursos de sua organização e, 
O emprego da análise de risco como ferramenta da inteligência estratégica

por conseguinte, mitigar seus riscos, ou pelo menos, minimizar aqueles riscos que são inaceitáveis do ponto de vista do processo decisório.

Conforme destacado neste trabalho, a AR pode ser realizada em diversos momentos, como no início de um novo planejamento de projeto ou como parte da gestão contínua e, ainda, como um estudo do que pode ocorrer após os riscos terem sido tratados. Entretanto, propõe-se, aqui, identificar sua importância para a inteligência estratégica, sobretudo quando da confecção de cenários prospectivos. ${ }^{4}$

\subsection{A APLiCABilidade da ANÁlise de Riscos EM CENÁRIOS PROSPECTIVOS}

O termo cenário assume variados significados, mas para nosso estudo, consideraremos o conceito que trata de combinações estatísticas de incertezas. Ou seja, cenário é o fundamento básico da prospectiva, onde os futuros são múltiplos e incertos.

Godet (1999, p. 60) apresenta uma definição mais abrangente. Entende que um cenário é "o conjunto formado pela descrição coerente de uma situação futura e pelo encaminhamento dos acontecimentos que permitem passar da situação de origem à situação futura”.

Existem diversas técnicas prospectivas utilizadas por diferentes países no mundo para definição de cenários, como por exemplo, Imagens de Futuro, Construção de Cenários, Técnicas Delphi, Técnicas de Impactos Cruzados, Técnicas de modelos de Simulação, Técnica de Exploração de Tendências, Técnicas de jogos de Simulação, e outras. Trataremos aqui da Técnica de Construção de Cenários Exploratórios Probabilísticos, realizada com o apoio de recursos de cálculo e de processamento de dados, adotado pela Escola Superior de Guerra (ESG) por meio da utilização do software denominado PUMA5.

Em se tratando dos Cenários Exploratórios Probabilísticos faz-se necessário destacar que estes se valem da conjugação de diversas técnicas para a

4 Cenário Prospectivo: é o conjunto formado pela descrição coerente de uma situação futura e pelo encaminhamento dos acontecimentos que permitem passar da situação de origem à situação futura, fundamentada em hipóteses coerentes sobre os prováveis comportamentos das variáveis determinantes do objeto de planejamento. (BRASIL, 2009, p. 21).

5 Sistema de Planejamento Estratégico e Cenários Prospectivos pela Brainstorming Assessoria de Planejamento e Informática Ltda. 
consecução de seu objetivo: o Brainstorming, a Delphi, os Impactos Cruzados, a Simulação de Monte Carlo e o Teorema de Bayes.

Como resultado da interação realizada pelo software PUMA, após a inserção de diversos dados, é obtido o Cenário mais provável, que apresenta a descrição da evolução da conjuntura atual até um futuro hipotético no horizonte temporal estabelecido, resultante da maior probabilidade de ocorrência, baseado nas opinióes de peritos. (BRAINSTORMING, 2010, p. 50).

A partir do cenário mais provável, o Decisor Estratégico escolhe a melhor conjugação de eventos segundo o interesse organizacional, baseando-se na seleção e levando em consideração os eventos favoráveis e não favoráveis, que é denominado cenário ideal. (BRAINSTORMING, 2010, p. 51).

Caso não ocorram rupturas de tendências, provavelmente ocorrerá o cenário de tendência, que é aquele que se equipara aos procedimentos realizados, de acordo com a previsão clássica, até o limite do horizonte temporal, de acordo com a percepção expressa pela equipe de planejamento, responsável pela elaboração dos cenários, denominada Grupo de Controle. (BRAINSTORMING, 2010, p. 50).

Nesse sentido, não resta dúvida que a técnica de Cenários Prospectivos é permeada a todo o momento pela atividade de inteligência, pois fornece informações relevantes para a construção desse cenário. Ainda, a técnica produz, através de metodologia própria e processos de raciocínio critico, a seleção e a organização das informações, além de facilitar a compreensão da realidade.

Depois de identificado os três cenários pelo PUMA, acima descritos, não há uma avaliação, por parte do programa, das consequências do cenário considerado pelo decisor como o Ideal. Ou seja, embora se definam quais são os eventos que deverão ou não ocorrer no cenário escolhido pelo tomador de decisão, não há um estudo especializado e técnico no que tange a identificação do impacto e das extensões que essa decisão pode ter. Essa questão será suprida pela ferramenta acessória da $\mathrm{AR}$ aqui proposta, uma vez que ao identificar os riscos e as possíveis ações para mitigá-lo, o decisor pode reavaliar todos os eventos do cenário.

Depreende-se, pois, que a AR é uma importante técnica acessória de inteligência no assessoramento estratégico ao processo decisório, uma vez que aumentará a qualidade técnica do resultado final apresentado. 
O emprego da análise de risco como ferramenta da inteligência estratégica

\section{Consideraçốes Finais}

A Análise de Risco mostra-se cada dia mais como uma forte tendência corporativa e institucional para aplicação no assessoramento estratégico aos tomadores de decisão, visando reduzir os riscos, identificar oportunidades e proporcionar segurança na execução de ações estratégicas.

Através da conjugação de diversas técnicas acessórias, é possível proporcionar maior segurança no planejamento e execução de processos levados a apreciação dos decisores, que orientem o planejamento de ações que reduzam o risco, ou que permitam a adoção de medidas que minimizem o dano que eventualmente venha a ser causado pelos eventos, durante o acompanhamento da ação em curso.

Um relatório de $A R$, quando elaborado pela inteligência, cumpre seu papel de assessoramento ao processo decisório, amparado em sólidos conhecimentos produzidos conforme a metodologia da produção do conhecimento. Sua apresentação permite distinguir entre diversas linhas de ação, não se prendendo a uma simples indicação, o que aumenta o seu valor consultivo.

A construção de cenários é um processo capaz de incrementar o processo decisório com informações dotadas de relevância, para agregar valores a organização. Esta técnica permite a visualização de imagem do futuro com diferentes ângulos de visão: o provável, o ideal e o de tendência, permitindo não somente a antecipação de eventos, mas a maximização da capacidade de identificação das ameaças e de oportunidades, reduzindo as incertezas na construção de um futuro resiliente.

A AR associada ao método de construção de cenários, quando realizada no contexto da atividade de inteligência no assessoramento ao processo decisório, atende a uma demanda atual das organizações, em que se faz mister que o decisor esteja melhor preparado para gerenciar e processar uma grande quantidade de informaçóes, analisar as ameaças e preparar-se para a prevenção e reação à sua ocorrência.

A AR promove o entendimento do nível de risco e de sua natureza, relacionado ao cenário ideal, aquele que foi escolhido pelo decisor. Esta análise permite calcular os respectivos impactos na organização, quer seja sobre o prisma financeiro, operacional, legal ou de sua própria imagem, permitindo 
que seja fundamentada a decisão do cenário Ideal para o gestor, tendo em vista que ele vislumbrará as possíveis consequências dessa decisão.

Desta maneira, parece apropriado considerar que a técnica de Cenários Prospectivos tem seu resultado maximizado pela permeação, a todo o momento, da atividade de inteligência, através da agregação de conhecimentos e informações relevantes, que foram produzidos por metodologia própria e processos de raciocínio crítico.

O emprego da inteligência na realização de análises de riscos, voltada para o assessoramento ao decisor na avaliação dos cenários produzidos por técnicas prospectivas, traz a vantagem da produção de conhecimento relevante e mais confiável, e permite a ampliação da visibilidade do risco inerente ao cenário em referência para a consecução do objetivo da organização e seu respectivo futuro, quer seja na esfera privada ou governamental.

No Brasil, com diversos obstáculos e inúmeras dificuldades ainda a serem superadas, é essencial o trabalho da atividade de inteligência com suas técnicas acessórias, como a Análise de Riscos.

Por fim, este trabalho sugere a possibilidade da aplicação conjunta de conceitos e suscita a necessidade de desdobramentos dessa pesquisa, tratando, especificamente, da aplicação dessa ferramenta ao âmbito da segurança pública.

Analista de InTeligênCIA Estratégica formado pela Escola Superior de Guerra. Papiloscopista Policial Federal, Especialista em Ciência Policial e Inteligência Policial pela Escola Superior de Polícia. Oficial Fuzileiro Naval da Reserva, Graduado em Ciências Navais pela Escola Naval (EN-1998). Professor da Academia Nacional de Polícia do Departamento de Polícia Federal (ANP/DPF) nos Cursos de Formação, Especialização e Aperfeiçoamento. Professor da Divisão de Doutrina de Inteligência Policial e Treinamento da Polícia Federal. Atualmente atuando na Área de Inteligência Estratégica, Inteligência Policial e Segurança Pública. P. 
O emprego da análise de risco como ferramenta da inteligência estratégica

Felipe Scarpelli de Andrade

ANALISTA DE INTELIGÊNCIA ESTRATÉGICA Formado pela EsCola

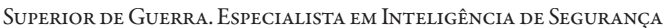
Pública pela Univesidade Sul de Santa Catarina. Agente de Polícia Federal. Professor da Academia Nacional de Polícia do Departamento de Polícia Federal (ANP/DPF) nos Cursos de FormaÇão, Especialização e Aperfeiçoamento. Professor da Doutrina de Inteligência (DINT) Da Polícia Federal nos cursos afetos À Área de Inteligência Policial. Conteudista da Academia Nacional de Polícia e Secretaria Nacional de Segurança Pública. Atualmente atuando na Área de InteligênCia Estratégica, Inteligência Policial e Segurança Pública..

E-MAIL: SCARPELLI.FSA@DPF.GOV.BR

\section{ABSTRACT}

\section{The Use of Risk Analysis as a Tool of Strategic Intelligence}

This work presents a proposal to improve advice to the decision-making process through the use of risk analysis as a tool for strategic intelligence, so coupled with prospective techniques, bringing a presentation of the current context and its applicability.

KEYwORDS: Intelligence. Risk analisys. Strategic intelligence.

\section{REFERÊNCIAS}

ABNT ISO GUIA 73:2009 - Gestão de riscos - Vocabulário. Rio de Janeiro: Associação Brasileira de Normas Técnicas, 2009.

BRAINSTORMING. Método Grumbach de Gestão Estratégica. Rio de Janeiro, 2010.

BRASIL. Lei nº 9883 de 7 de dezembro de 1999. Institui o Sistema Brasileiro de Inteligência, cria a Agência Brasileira de Inteligência - Abin, e dá outras providências. Diário Oficial da República Federativa do Brasil. Disponível em: <http:// www.abin.gov.br/modules/mastop_publish/?tac=Lei_9883_ de_07\%2F12\%2F1999>. Acesso em: 10 de jun. de 2013.

. Decreto 4376/2002, de 13 de setembro de 2002. Dispõe sobre a organização e o funcionamento do Sistema Brasileiro de Inteligência, e dá outras providências. Diário Oficial da República Federativa do Brasil. Disponível em: <http://www.abin.gov.br/modules/ mastop_publish/?tac=Decreto_4376\%2F2002 >. Acesso em: 15 de 
jun. de 2013.

CEPIK, Marco A. C. Serviços de inteligência: agilidade e transparência como dilemas de institucionalização. Rio de Janeiro, IUPERJ, 2001.

DRUCKER, Peter. Administração Lucrativa. Rio de Janeiro: Editora Zahar, 1972.

ESCOLA SUPERIOR DE GUERRA (Brasil). Método para o

Planejamento Estratégico/ESG. Rio de Janeiro. 2009.

ESTADOS UNIDOS DA AMÉRICA (EUA). Homeland Security Risk Management Doctrine. Washington DC, DHS, 2011. Disponível em: $<$ http://www.dhs.gov/xlibrary/assets/rma-risk-managementfundamentals.pdf $>$. Acesso em 08 jun. 2013.

\section{ESTADOS UNIDOS DA AMÉRICA (EUA). The National} Counterintelligence Strategy of the United States of America. Washington DC, NCIX, 2007. Disponível em: <http://www.ncix. gov/publications/strategy/docs/CIStrategy.pdf $>$. Acesso em 10 jun. 2013.

FERRO JÚNIOR, C. M.; ALVES, C. F.; MORESI, E. A. D.; NEHME, C. C. Cognição Organizacional: Um Estudo da Tecnologia da Informação Aplicada à Análise de Vínculos na Atividade Policial. In: Anais do Congresso Brasileiro de Gestão do Conhecimento KM Brasil 2008.

GODET, M. From Anticipation to Action. Unesco, Paris, 1999.

RIBEIRO, Antonio de Lima. Teorias da Administração. São Paulo: Editora Saraiva, 2003.

SIMON, Herbert Alexander. A capacidade de decisão e liderança. Rio de Janeiro: Fundo de Cultura, 1963.

VALENTIM, M. L. P. (Org.). Gestão da informação e do conhecimento no âmbito da Ciência da Informação. São Paulo: Polis: Cultura Acadêmica, 2008.

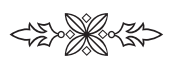

